\title{
SEPARABILITY OF STOCHASTIC PROCESSES
}

\author{
S. H. COLEMAN ${ }^{1}$
}

This paper applies a generalization of the Daniell theory of integration to stochastic processes with values in a compact Hausdorff space. A consistent family of integrals on continuous functions of a finite number of variables is extended by the procedure (developed by McShane and Bourbaki) outlined in $\$ 1$ to an integral on functions defined on an arbitrary product of compact Hausdorff spaces. \$2 proves, in a generalized form suited to processes with values in a compact metric space, that such a process is always separable. The applications given there are intended to be illustrative rather than comprehensive; one or two may be novel. In $\S 3$, it is shown that the measure resulting from this procedure is a regular Borel measure, so that the results of Nelson [5] on such measures hold. The characterization of the measures of open and of closed sets given there seems to be new. Approximations to integrable functions are discussed in $\$ 4$, and a theorem of Doob [3] on the approximation of certain sets by others depending only on coordinates belonging to an arbitrary dense parameter set is generalized to include all integrable functions. The question of measurability of processes will not be discussed except to mention here that the usual counterexamples to measurability [2] no longer hold, while the known results on measurability without modification of the measure $[3 ; 5]$ remain valid.

1. The integral. The integration theory used here can be found in detail in $[1 ; 4 ; 6]$.

A set of elementary functions on a set $\Omega$ is a vector lattice $\mathcal{E}$ of realvalued functions on $\Omega$. An elementary integral on $\varepsilon$ is a linear functional $I$ on $\varepsilon$ satisfying $I(|e|) \geqq 0$ and the continuity condition: if $S \subset \mathcal{E}$ is directed by $\geqq$, if $e_{0} \in \mathcal{E}$ and if $\sup \{e: e \in S\} \geqq e_{0}$, then $\sup \{I(e): e \in S\} \geqq I\left(e_{0}\right)$. An extended-real-valued function $u$ on $\Omega$ is a $U$-function if there is a set $S$, directed by $\geqq$, of elementary functions such that $u=\sup \{e: e \in S\}$, and $I$ can then be extended to $u$ by setting $I(u)=\sup \{I(e): e \in S\}$.

If $f$ is any extended-real-valued function on $\Omega$, define $N(f)$ by setting $N(g)=\inf \{I(u): u$ a $U$-function, $u \geqq|f|\}$. Then the set $\mathcal{F}$ of

Presented to the Society, January 29, 1960 under the title $A$ Daniell approach to stochastic processes; received by the editors December 28, 1961.

${ }^{1}$ Supported in part by National Science Foundation Grant G-14362. This work is based on the author's doctoral dissertation written at the University of Virginia under the direction of Professor E. J. McShane. 
equivalence classes of functions $f$ with $N(f)<\infty$, modulo functions $g$ with $N(f)=0$, is a complete normed linear space with norm $N . I$ is continuous in this norm and therefore extends to the completion, the class of integrable functions, of $\mathcal{E}$ in $\mathcal{F}$. Measurable functions and sets can then be defined, and if $\Omega$ itself is a measurable set, the set function $m$, defined by setting $m(A)=I(f)$ if the characteristic function $f$ of $A \subset \Omega$ is integrable, is a measure.

McShane [4] has proved for the class of $U$-functions a generalization of the monotone convergence theorem which will be needed.

TheOREM 1.1. If a set $S$ of $U$-functions is directed by $\geqq$ and if $I$ is bounded on $S$, then $u_{0}=\sup \{u: u \in S\}$ is an integrable $U$-function, and $I\left(u_{0}\right)=\sup \{I(u): u \in S\}$.

If $X$ is a compact Hausdorff space and $T$ a parameter set, let $\Omega=X^{T}$ be the set of all functions from $T$ into $X$, topologized by the Tychonov topology. A real-valued function $f$ on $\Omega$ is based on $S \subset T$ if $f(\omega)=f\left(\omega^{\prime}\right)$ whenever $\omega(s)=\omega^{\prime}(s)$ for all $s \in S$. A function on $\Omega$ is finitely (countably) based if it is based on a finite (countable) subset of $T$. The set $\mathcal{E}$ of all finitely based real-valued continuous functions on $\Omega$ is clearly a set of elementary functions. Suppose that, for each finite subset $A$ of $T, I_{A}$ is an integral on the continuous functions based on $A$ with $I_{A}(1)=1$. If this collection of integrals satisfies the consistency condition that $I_{A}(e)=I_{B}(e)$ whenever $A \subset B \subset T$ with $B$ finite and $e$ an elementary function based on $A$, then a non-negative linear functional $I$ can be defined on $\varepsilon$ by setting $I(e)=I_{A}(e)$ if $e$ is based on $A$. Since $\Omega$ is compact, Dini's theorem shows that $I$ satisfies the continuity condition above and is therefore an elementary integral. The measure $P$ induced by $I$ is a probability measure, and $\Omega$ with $P$ is a stochastic process of function-space type.

A modification of the usual argument proves the following fundamental lemma.

LEMMA 1.2. A function $u$ on $\Omega$ is a $U$-function if and only if $u$ is lower semi-continuous and bounded below.

Proof. Necessity follows from the definition of a $U$-function. To prove sufficiency, let $\omega \in \Omega$ and let $\epsilon$ be positive. Using lower semicontinuity of $u$ and the definition of the product topology, there is a finitely based open set $V$ containing $\omega$ such that $u\left(\omega^{\prime}\right)>u(\omega)-\epsilon$ for all $\omega^{\prime} \in V$. Applying Urysohn's lemma to the cartesian product of $X$ with itself over the finite base of $V$, there is a finitely based continuous function $e$ such that $e \leqq u$ and $e(\omega)>u(\omega)-\epsilon$. Therefore $u$ is the supremum of the directed set of all elementary functions below it, completing the proof. 
2. Separability. For simplicity, our main theorem will be stated and proved only for functions of two variables, although it clearly holds for functions of any finite number of variables.

TheOREM 2.1. Let $f$ be a lower semi-continuous function on $X \times X$. Let $T_{1}$ be a subset of $T$, and for each $t \in T_{1}$, let $S(t)$ be a subset of $T$. Then the function $F$ defined for each $\omega \in \Omega$ by

$$
F(\omega)=\sup _{s \in S(t), t \in T_{1}} f(\omega(s), \omega(t))
$$

is measurable and equal almost everywhere to the corresponding supremum over some countable subset of the union of $T_{1}$ and the $S(t) . A$ dual theorem holds for upper semi-continuous functions on $X \times X$.

Proof. It may be assumed that $f$ is bounded. Let $D$ be the collection of subsets $\Delta=\left\{t_{1}, \cdots, t_{n}, S_{\Delta}\left(t_{1}\right), \cdots, S_{\Delta}\left(t_{n}\right)\right\}$ of $T$, each consisting of a finite number of points $t_{1}, \cdots, t_{n}$ of $T_{1}$ and of finite subsets $S_{\Delta}\left(t_{j}\right)$ of $S\left(t_{j}\right), j=1, \cdots, n$. For each $\Delta$ in $D$, define $F_{\Delta}$ by setting, for each $\omega \in \Omega$,

$$
F_{\Delta}(\omega)=\sup _{t_{i} \in \Delta, s_{j} \in S_{\Delta}\left(t_{i}\right)} f\left(\omega\left(t_{i}\right), \omega\left(s_{j}\right)\right) .
$$

Then each $F_{\Delta}$ is a finitely-based $U$-function, and the collection $\left\{F_{\Delta}: \Delta \in \mathbb{D}\right\}$ is directed by $\geqq$. Since $f$ is bounded, $\left\{I\left(F_{\Delta}\right): \Delta \in \mathbb{D}\right\}$ is a bounded set, and therefore by Theorem 1.1, $F=\sup \left\{F_{\Delta}: \Delta \in \mathbb{D}\right\}$ is integrable and $I(F)=\sup \left\{I\left(F_{\Delta}\right): \Delta \in \mathbb{D}\right\}$.

There exists a monotone nondecreasing sequence $\left\{F_{\Delta_{n}}\right\}$ in $\left\{F_{\Delta}: \Delta \in \mathbb{D}\right\}$ such that $F^{\prime}=\sup F_{\Delta_{n}}$ is equal to $F$ almost everywhere, and

$$
F^{\prime}(\omega)=\sup _{s \in S^{\prime}(t), t \in T_{\mathrm{i}}^{\prime}} f(\omega(s), \omega(t))
$$

where $T_{1}^{\prime}$ is countable and each $S^{\prime}(t)$ is countable, completing the proof.

Suppose $T$ and $X$ are compact metric spaces, and let $d$ denote the metric on both. Then $d$ is continuous on $X \times X$, so that Theorem 2.1 applies. Standard separability arguments then show the measurability of such subsets of $\Omega$ as the continuous functions and the bounded functions. Some examples of other applications follow.

I. If $\alpha$ is positive and $s \neq t$, the function defined on $X \times X$ by $[d(x, y)][d(s, t)]^{-\alpha}$ is continuous. Therefore the function $F$ defined for each $\omega \in \Omega$ by

$$
F(\omega)=\sup _{s \neq t}[d(\omega(s), \omega(t))][d(s, t)]^{-\alpha}
$$


is measurable. It follows that $\cup_{n=0}^{\infty}\{\omega: F(\omega) \leqq n\}$, the set of functions from $T$ to $X$ which satisfy a Hölder condition of exponent $\alpha$, is measurable.

II. If $T$ is a real interval and $t$ is interior to $T$, the function $f_{t, n}$ defined by

$$
f_{t, n}(\omega)=\sup _{\{s: s<t, d(s, t)<1 / n)} d(\omega(s), \omega(t))
$$

is measurable. Then $f_{t}=\lim _{n} f_{t, n}$, the left oscillation of $\omega$ at $t$, is measurable. In particular, the set of $\omega$ with left limits at $t$ is measurable.

III. If $X$ and $T$ are real intervals, the total variation $V(\omega)$ of $\omega$ over $T$ is lower semi-continuous and therefore measurable. Thus the set $\{\omega:|V(\omega)|<\infty\}$, the set of functions of bounded variation, is measurable.

IV. Suppose $T$ and $X$ are real intervals. For each $m$, let $A_{m}$ be the set of finite collections $\alpha$ of disjoint intervals $\left\{\left(s_{1}, t_{1}\right), \cdots,\left(s_{j \alpha}, t_{j \alpha}\right)\right\}$ of $T$ with $\sum_{1}^{j \alpha}\left(t_{k}-s_{k}\right)<1 / m$. Then $f_{m}$ defined by

$$
f_{m}(\omega)=\sup _{\alpha \in A_{m}} \sum_{k=1}^{j \alpha}\left|\omega\left(t_{k}\right)-\omega\left(s_{k}\right)\right|
$$

is lower semi-continuous, and the set $S_{m, n}=\left\{\omega: f_{m}(\omega)<1 / n\right\}$ is measurable. Then $S=\bigcap_{n=1}^{\infty} \cup_{m=1}^{\infty} S_{m, n}$, the set of absolutely continuous functions from $T$ into $X$, is measurable.

\section{The measure.}

TheOREM 3.1. The measure $P$ defined by $I$ on subsets of $\Omega$ is a regular Borel measure.

Proof. The characteristic function of a closed set is upper semicontinuous and therefore measurable. Regularity is proved by the standard argument as in Bourbaki [1, p. 156].

THEOREM 3.2. The measure of an open set $G$ is the supremum of the measures of all finitely based open sets contained in it. The measure of a closed set $F$ is the infimum of the measures of all finitely-based closed sets containing it.

Proof. If $G$ is open, its characteristic function is lower semicontinuous. Given $\epsilon>0$, there is an elementary function $e$ with $e \leqq \chi G$ and $I(e)>P(G)-\epsilon$. The set $G^{\prime}=\{\omega: e(\omega)>0\}$ is open, finitely-based and contained in $G$, and $P\left(G^{\prime}\right) \geqq I(e) \geqq P(G)-\epsilon$. The proof for a closed set is dual.

Corollary 3.3. An open set, $a G_{\delta}, a G_{\delta \sigma}, \cdots$ is equivalent to $a$ 
countably-based open set, $G_{\delta}, G_{\delta \sigma}, \cdots$ contained in it. A closed set, an $F_{\sigma}$, an $F_{\sigma \delta}, \cdots$ is equivalent to a countably-based closed set, $F_{\sigma}$, $F_{\sigma \delta}, \cdots$ containing it.

4. Approximation of integrable functions. If $f$ is any integrable function, it is the limit almost everywhere of a sequence of elementary functions and is therefore equivalent to a countably-based Baire function. (We remark that the density, in the uniform norm, of elementary functions in the set of continuous functions implies that every Baire function on $\Omega$ is countably based.) If $T$ and $X$ are metric and the process is continuous in probability, that is, if it satisfies

$$
\lim _{d(s, t) \rightarrow 0} P\{\omega: d(\omega(s), \omega(t)) \geqq \epsilon\}=0
$$

for every $t \in T$ and $\epsilon>0$, this result can be improved as follows.

THEOREM 4.1. If $X$ and T are metric and the stochastic process defined by $P$ is continuous in probability, then for every countable dense subset $S$ of $T$ and every integrable function $f$, there is an integrable function $f^{\prime}$ based on $S$ which is equal almost everywhere to $f$.

Proof. Suppose $e$ is elementary and $e(\omega)=e\left(\omega\left(t_{1}\right), \cdots, \omega\left(t_{n}\right)\right)$. Using continuity and boundedness of $e$ and continuity in probability of the process, there is, for every positive $\epsilon$, a set $\left\{t_{1}^{\prime}, \cdots, t_{n}^{\prime}\right\}$ contained in $S$ such that if $e^{\prime}$ is the elementary function defined by $e^{\prime}(\omega)=e\left(\omega\left(t_{1}^{\prime}\right), \cdots, \omega\left(t_{n}^{\prime}\right)\right)$, then $I\left(\left|e-e^{\prime}\right|\right)<\epsilon$. Then if $f$ is integrable and $\lim I\left(\left|f-e_{n}\right|\right)=0$, there is a sequence $\left\{e_{n}^{\prime}\right\}$ of elementary functions based on $S$ with $\lim I\left(\left|f-e_{n}^{\prime}\right|\right)=0$, and a subsequence converges to a function $f^{\prime}$ based on $S$ and equal almost everywhere to $f$.

\section{Bibliography}

1. N. Bourbaki, Intégration, Actualités Sci. Ind. No. 1175, Hermann, Paris, 1952.

2. J. L. Doob, Probability in function space, Bull. Amer. Math. Soc. 53 (1947), 15-30.

3. - Stochastic processes, Wiley, New York, 1953.

4. E. J. McShane, Remark concerning integration, Proc. Nat. Acad. Sci. U.S.A. 35 (1949), 46-49.

5. E. Nelson, Regular probability measures on function space, Ann. of Math. (2) 69 (1959), 630-643. 50-58.

6. M. H. Stone, Notes on integration. IV, Proc. Nat. Acad. Sci. U.S.A. 35 (1949),

UNIVERSITY OF WISCONSIN 